\title{
MRI-based nomogram analysis: recognition of anterior peritoneal reflection and its relationship to rectal cancers
}

Shaoting Zhang ${ }^{1}$, Fangying Chen ${ }^{1}$, Xiaolu Ma ${ }^{1}$, Minjie Wang ${ }^{1}$, Guanyu Yu ${ }^{2}$, Fu Shen ${ }^{1^{*}+} \mathbb{D}$, Xianhua Gao ${ }^{2^{*}+}$ and Jianping Lu ${ }^{1 * \dagger}$

\begin{abstract}
Background: This study is aimed to explore the factors influencing the visualization of the anterior peritoneal reflection (APR) and evaluated the feasibility of measuring the distance from the anal verge to APR (AV-APR), the tumor height on MRI and the accuracy of determining the tumor location with regard to APR.

Methods: We retrospectively analyzed 110 patients with rectal cancer. A univariate and multivariate logistic regression was performed to identify the independent factors (age, sex, T stage, the degree of bladder filling, pelvic effusion, intraoperative tumor location, $\mathrm{BMI}$, uterine orientation, the distance from seminal vesicle/uterus to rectum) associated with the visualization of the APR on MRI. The nomogram diagram and receiver operating characteristic curve (ROC curve) were established. Intraclass correlation coefficient (ICC) was used to evaluate the consistency of the distance of AV-APR. The Pearson correlation coefficient was used to characterize the agreement between measurements of the tumor height by colonoscopy and MRI. The Kappa statistics was used to evaluate the value of MRI in the diagnosis of the tumor location with regard to the APR.

Results: Multivariate logistic regression showed that $\mathrm{BMI}(P=0.031$, odds ratio, $\mathrm{OR}=1.197)$, pelvic effusion $(P=0.020$, $\mathrm{OR}=7.107)$ and the distance from seminal vesicle/uterus to the rectum $(P=0.001, \mathrm{OR}=3.622)$ were correlated with the visualization of APR. The cut-off point of $\mathrm{BMI}$ and the distance from seminal vesicle/uterus to the rectum is $25.845 \mathrm{~kg} / \mathrm{m}^{2}$ and $1.15 \mathrm{~cm}$. The area under curve (AUC) (95\% Confidence Interval, 95\% Cl) of the combined model is $0.840(0.750-0.930)$. The favorable calibration of the nomogram showed a non-significant Hosmer-Lemeshow test statistic $(P=0.195)$. The ICC value $(95 \% \mathrm{Cl})$ of the distance of AV-APR measured by two radiologists was $0.981(0.969-$ 0.989). The height measured by MRI and colonoscopy were correlated with each other $(r=0.699, P<0.001)$. The Kappa value was 0.854 .

Conclusions: BMI, pelvic effusion, and the distance from seminal vesicle/uterus to rectum could affect the visualization of APR on MRI. Also, it's feasible to measure the distance of AV-APR, the tumor height, and to evaluate the tumor location with regard to APR using MRI.
\end{abstract}

\footnotetext{
*Correspondence: ssff_53@163.com; gxh505@126.com; cjr.

lujianping@vip.163.com

${ }^{\dagger} \mathrm{Fu}$ Shen, Xianhua Gao and Jianping Lu contributed equally to the study

and share corresponding authorship

${ }^{1}$ Department of Radiology, Changhai Hospital, 168 Changhai Road,

Shanghai 200433, China

2 Department of Colorectal Surgery, Changhai Hospital, 168 Changhai

Road, Shanghai 200433, China
}

(C) The Author(s) 2021. Open Access This article is licensed under a Creative Commons Attribution 4.0 International License, which permits use, sharing, adaptation, distribution and reproduction in any medium or format, as long as you give appropriate credit to the original author(s) and the source, provide a link to the Creative Commons licence, and indicate if changes were made. The images or other third party material in this article are included in the article's Creative Commons licence, unless indicated otherwise in a credit line to the material. If material is not included in the article's Creative Commons licence and your intended use is not permitted by statutory regulation or exceeds the permitted use, you will need to obtain permission directly from the copyright holder. To view a copy of this licence, visit http://creativecommons.org/licenses/by/4.0/. The Creative Commons Public Domain Dedication waiver (http://creativeco mmons.org/publicdomain/zero/1.0/) applies to the data made available in this article, unless otherwise stated in a credit line to the data. 
Keywords: Magnetic resonance imaging, Anterior peritoneal reflection, Rectal cancer, Nomogram

\section{Background}

Colorectal cancer $(\mathrm{CRC})$ is one of the most common gastrointestinal cancers, with rectal cancer $(\mathrm{RC})$ accounting for $30-35 \%$. According to the reported data, CRC ranks third in incidence and second in mortality worldwide [1]. Over the past decade, the incidence of CRC has rapidly declined in the wake of widespread colonoscopy uptake in developed countries. However, the decline in the overall CRC incidence rate masked an increase of $2 \%$ per year among adults younger than 55 years that have been recorded over recent years [2,3]. The peritoneum covers the anterior wall of the upper rectum, whereas the middle and lower thirds lie below the peritoneal reflection and are completely encircled by mesorectum [4]. Above and below the anterior peritoneal reflection (APR), the lymphatic spread of cancer is inconsistent $[5,6]$. Under the APR, they are mainly drained through the lateral lymph, while above the APR, they are mainly drained to the inferior mesentery. And according to the location and stage of the tumor, the treatment and prognosis of rectal cancer may significantly differ $[7,8]$. Some surgeons propose that the APR could be a suitable landmark for identifying patients with rectal cancer for radiation. And the overall reported 5-year local recurrence rate for intraperitoneal and extraperitoneal rectal cancer is $4.2 \%$ and $13.3 \%$, respectively [9]. Therefore, accurate recognition of the APR and the tumor location with regard to APR before the operation is useful in choosing the appropriate treatment strategies so as to avoid under or overtreatment [10-14]. In previous studies, rigid endoscopy or intraoperative proctoscopy were used to measure the distance of AV-APR $[15,16]$. Still, the results were highly variable, and the tumor location towards the APR could not be accurately evaluated.

Magnetic resonance imaging (MRI) has a good soft tissue resolution and can also identify APR [17-19]. On the axial T2-weighted (T2W) images, APR shows a V-shaped hypointense configuration attached to the anterior rectal wall. However, the axial images cannot precisely reveal the distance of AV-APR. On the sagittal T2W images, APR was identified as a thin hypointense linear structure noted along with the superior bladder (men) or uterus (women), which extended inferiorly and posteriorly to the tip of the seminal vesicles in men and to the cul-de-sac in women, after which the posterior extension attached to the anterior rectal wall (Fig. 1a, b) [20]. Unfortunately, not all APRs can be visualized on MRI in clinical practice [4].

Therefore, we explored the factors that affect the visualization of the APR on sagittal MRI. Moreover, we assessed the feasibility of measuring the distance of AVAPR and the height of the tumor using MRI, as well as the accuracy of MRI for recognizing the tumor location with regard to the APR.

\section{Methods \\ Patients}

All methods of the present research were carried out in accordance with the Declaration of Helsinki and were approved by the local Institutional Review Board

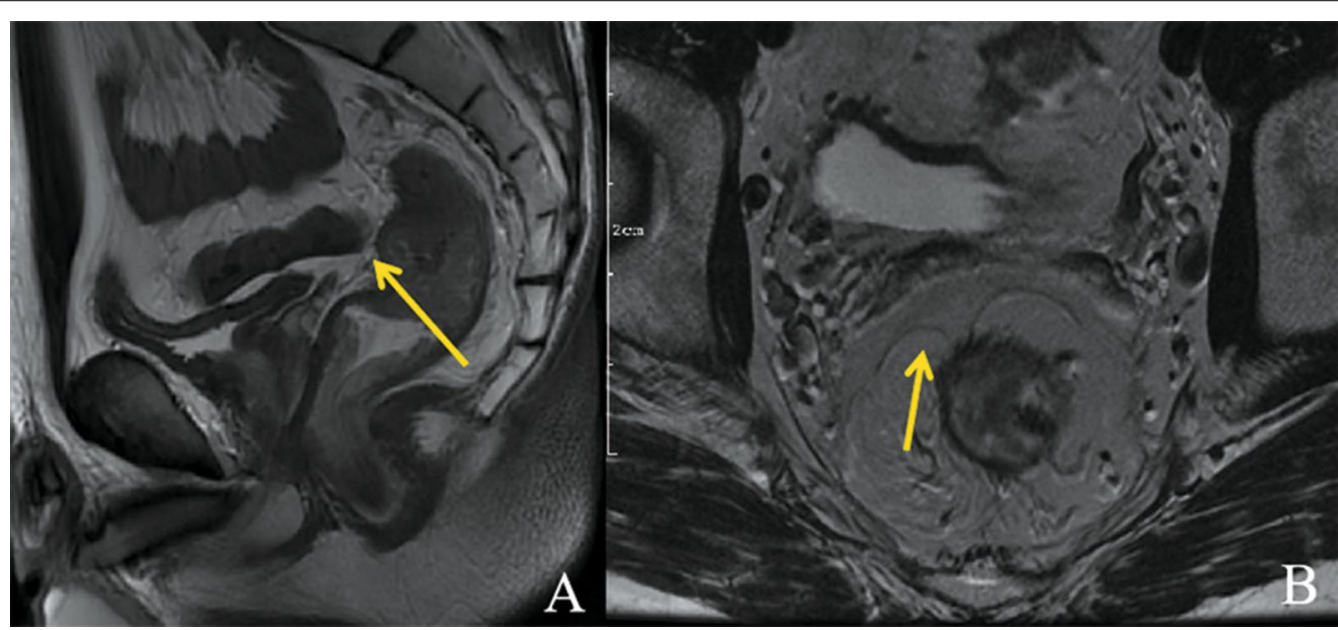

Fig. 1 Identification of the APR on MR imaging. a Sagittal plane: the APR was a thin hypointense linear structure noted along with the superior bladder, which extended inferiorly and posteriorly to the tip of the seminal vesicles, after which the posterior extension attached to the anterior rectal wall. b Axial plane: the APR attaches to the anterior rectal wall in a V-shaped hypointense configuration. APR: anterior peritoneal reflection 


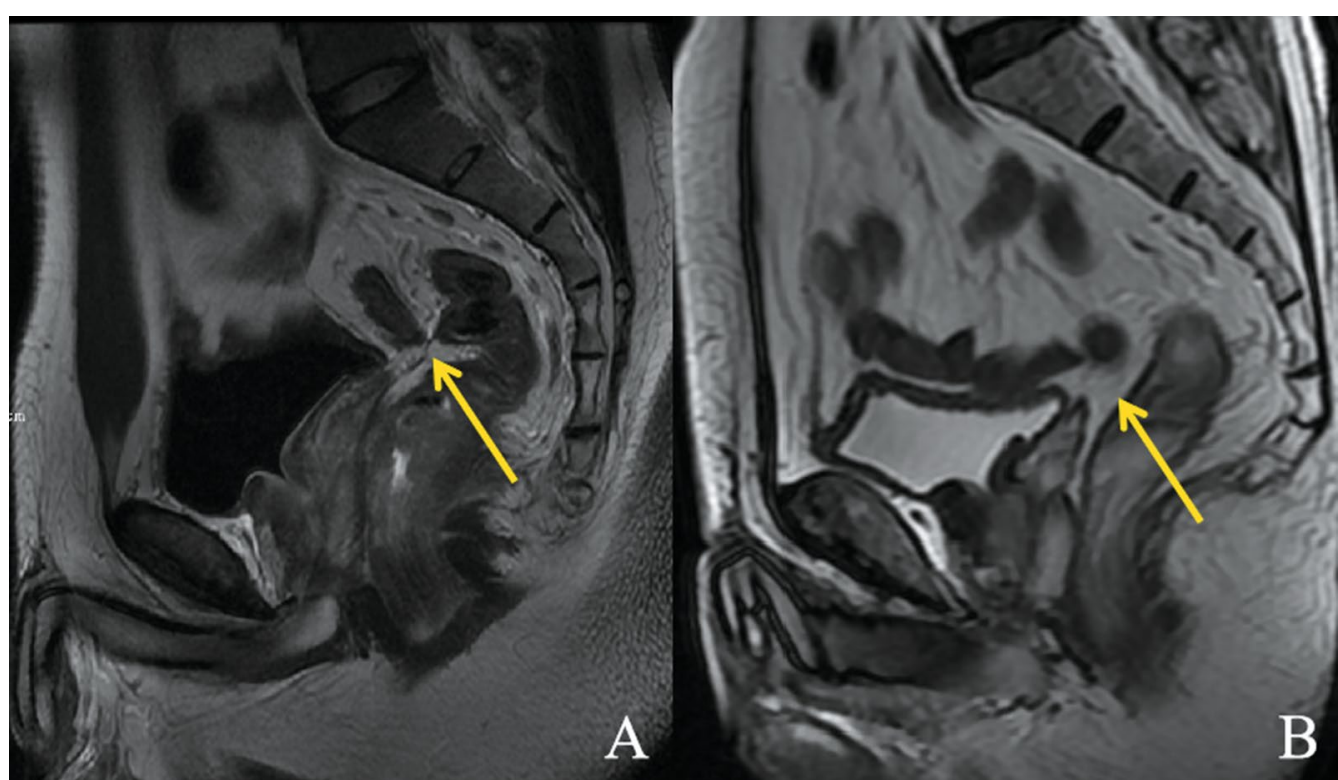

Fig. 2 The visualization of the APR on sagittal T2W images. a APR was definitely visible. b APR was probably visible

(Committee on Ethics of Biomedicine, Changhai Hospital, Shanghai, China). Informed consent was waived for this retrospective study. A total of 320 consecutive patients diagnosed with rectal cancer and then treated in our hospital between January 2019 and June 2019 were enrolled in this retrospective study. Selection criteria included the following: (1) a biopsy-confirmed primary rectal carcinoma, (2) treatment by surgical resection, (3) a preoperative rectal MRI with good image quality, (4) operation within two weeks after MRI. Exclusion criteria included the following: (1) patients with MRI images with motion artifacts or poor image quality $(n=30)$, (2) patients who received radiotherapy, neoadjuvant treatment or/and palliative treatment $(\mathrm{n}=100)$, (3) an interval between MRI and surgery higher than 2 weeks $(n=20)$, (4) patients with the previous history of other pelvic surgery $(n=60)$.

The collected clinical and imaging data included the following: patient age, sex, Body Mass Index (BMI), $\mathrm{T}$ stage, the tumor location with regard to APR (MRI and intraoperative findings), the degree of bladder filling, the orientation of the uterus, pelvic effusion, and the distance from seminal vesicle/uterus to the rectum, the height of tumor (MRI and colonoscopy), and the distance of AV-APR.

\section{MRI-sequence acquisition}

MRI was performed on a 3.0 Tesla (T) MRI scanner (MAGNETOM Skyra, Siemens Healthcare, Erlangen, Germany) using a phased-array body coil while patients were placed in a supine position. Before scanning, intestinal cleaning was performed by enema administration with $20 \mathrm{ml}$ of glycerin. MRI scan sequences included: (1) high-resolution oblique axial T2WI without fat saturation, (2) sagittal T2WI (turbo-spin-echo, TSE) without fat saturation, (3) axial T1-weighted image (T1WI), (4) axial diffusion weighted images $\left(b=0,1000 \mathrm{~s} / \mathrm{mm}^{2}\right)$, and (5) gadolinium contrast-enhanced T1WI with fat saturation (axial, sagittal and coronal planes). The scanning parameters of sagittal T2WI were as follows: repetition time/ echo time [TR/TE]: 5000/106 ms, field of view [FOV]: $23 \mathrm{~cm}$, section thickness: $5 \mathrm{~mm}$, number of slices: 23 slices, voxel: $0.7 * 0.7 * 5.0 \mathrm{~mm}$, bandwidth: $200 \mathrm{~Hz} /$ pixel, averages: 2 , flip angle: 180 , total time: $157 \mathrm{~s}$.

\section{Radiologist and colorectal surgeons revaluation strategy and anatomic measurements Radiologists}

MR images were independently reviewed by two gastrointestinal radiologists (SZ and FC) with more than 5 years' working experience in rectal MRI. The two readers were blinded to any pathological results of the patients and achieved a unified standard to evaluate the MRI features through discussion before the independent analysis. If there were discrepancies between the two readers on qualitative parameters, a final decision was reached by a third reader (FS with 11 years of experience in imaging diagnosis). The readers cross-referenced the oblique axial T2W and sagittal $\mathrm{T} 2 \mathrm{~W}$ imaging to recognize APR. We divided APR into two categories: definitely 
visible (the thin hypointense linear structure attached to the anterior rectal wall is definitely visible) and probably visible (the thin hypointense linear structure is probably visible or definitely not visible) (Fig. 2a, b) [19]. For all the patients rated with a definitely visible APR, the distances of AV-APR were measured in the sagittal T2W images as a line from the APR to the anal verge along the direction of the rectum (Fig. 3a) [4]. For all the patients, the height of tumor was measured as the distance from the inferior tumor margin to the anal verge (Fig. 3b). The distances from seminal vesicle/uterus to rectum were measured as a line from the junction of APR and seminal vesicle/uterus to the junction of APR and rectum along the direction of APR (Fig. 3c). The tumor location with regard to APR (MRI) was assigned to the following categories: (1) above the APR (the distal end of the tumor reaches above the height of the APR), (2) straddle the APR (the distal end of the tumor reaches below the height of the APR and the proximal end of the tumor reaches above the height of the APR), (3) below the APR (the proximal end of the tumor reaches below the height of the APR). According to the angle between the axis of the uterus and the axis of the axial plane on the sagittal T2WI, uterine orientation was categorized as follows: anteversion, perpendicular, or retroversion. A distended bladder was defined as the bladder wall showing no folds on both sagittal and axial T2WI [4], which means that the bladder was filling.

\section{Colorectal surgeons}

The two surgeons were blinded to any MRI results of the patients. For all the patients, the height of tumor was measured by colonoscopy. And the tumor location with regard to APR (intraoperative findings) was also assigned to the following categories: (1) above the APR, (2) straddle the APR, (3) below the APR. If there were discrepancies between the two reviewers, a final decision was reached by consensus.

\section{Statistical analysis}

For continuous variables, the normality test was carried out by One-Sample Kolmogorov-Smirnov test. Data conforming to normal distribution were reported as mean \pm standard deviation, and data not conforming to normal distribution were reported as median and quartile. The Chi-square test or Fisher's exact test was carried out for assessing categorical variables, as appropriate. The Independent-Samples $\mathrm{T}$ test was carried out for assessing continuous variables. A univariate logistic regression analysis was performed to identify the independent factors associated with the visualization of the APR on MRI. Then, multivariate logistic regression combined the selected influencing factors was performed to establish a combined model. Moreover, the nomogram analysis and the receiver operating characteristic curve (ROC curve) were performed. Intraclass correlation coefficients (ICC) were used to evaluate the differences in the distance of AV-APR between the two radiologists. The Pearson correlation coefficient was used to characterize the agreement between pairs of measurements of the tumor height by colonoscopy and MRI. The consistency check of a diagnostic test (Kappa statistics) was used to evaluate the value of MRI in the diagnosis of the tumor location with regard to the APR. Statistical analyses were performed using SPSS version 19.0 for windows (SPSS Inc., Chicago, Illinois, USA) and $\mathrm{R}$ software (version

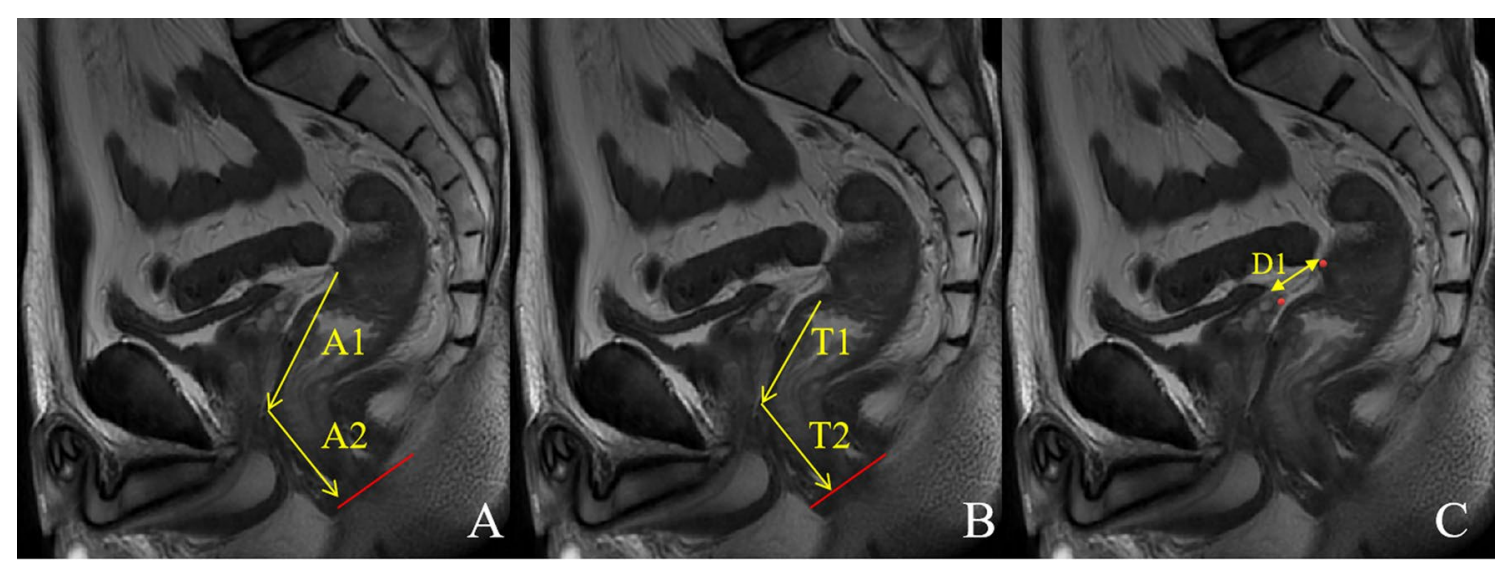

Fig. 3 Measurement of various distances on sagittal T2W images. a The height of the APR from the anal verge as a line from the APR to the anal verge along the direction of the rectum: $\mathrm{A} 1+\mathrm{A} 2$. $\mathbf{b}$ The tumor height from the anal verge was measured as the distance from the inferior tumor margin to the anal verge: $T 1+T 2$. $\mathbf{c}$ The distance from the seminal vesicle to the rectum was measured as a line from the junction of APR and seminal vesicle to the junction of APR and rectum along the direction of APR: D1 
3.4.3). All $P$ values $<0.05$ were considered statistically significant.

\section{Results}

\section{Clinical characteristics}

A total of 110 patients were finally included in this study. The APR was "definitely visible" in 75 of 110 cases (68.2\%) and "probably visible" in 35 of 110 cases (31.8\%) (definitely not visible was 0 ). The tumor height measured by MRI, age, BMI, the distance of AV-APR conformed to normal distribution $(P>0.05)$, and the tumor height measured by colonoscopy, the distance from seminal vesicle/uterus to rectum didn't conform to normal distribution $(P<0.05)$. The mean age was $60.22 \pm 10.03$ (range, 35-85) years. Other characteristics of patients are listed in Table 1. The two observers' agreement for objective parameters are listed in Additional file 1: Supplemental Table 1.
Distance of AV- APR measured upon MRI and comparisons of tumor height measured by colonoscopy and MRI

There were 75 patients with definitely visible APRs, including 32 females and 43 males. The mean distance of AV-APR was $9.60 \pm 1.22 \mathrm{~cm}$ in total, $9.57 \pm 0.68 \mathrm{~cm}$ in females, $9.62 \pm 1.47 \mathrm{~cm}$ in males $(P=0.857)$. The ICC value (95\% Confidence Interval, 95\% CI) of the distance measured by two radiologists was 0.981 (0.969-0.989). The mean height of the tumor measured by MRI was $6.89 \pm 2.53 \mathrm{~cm}$. The median and interquartile range of the height measured by MRI and colonoscopy were 7.05 $(5.21-8.75)$ and $7.00(5.00-10.00) \mathrm{cm}$, respectively. The two results were correlated with each other $(r=0.699$, $P<0.001)$.

\section{Comparisons of tumor location with regard to the APR by MRI and by intraoperative findings}

The accuracy of the tumor locations with regard to the APR (determined via MRI) was $90.0 \%$ compared with intraoperative findings (Fig. 4, Table 2). The Kappa value of tumor location with respect to the APR determined by MRI and intraoperative findings was $0.854(P<0.001)$.

Table 1 Characteristics of patients with rectal cancer

\begin{tabular}{|c|c|c|c|c|}
\hline \multirow[t]{2}{*}{ Variables } & \multicolumn{3}{|c|}{ No. of case/mean $\pm \mathrm{SD} /$ median (interquartile range) } & \multirow[t]{2}{*}{$P$ value } \\
\hline & Total $(\mathrm{N}=110)$ & $\begin{array}{l}\text { APR with probably visible } \\
(\mathrm{N}=35)\end{array}$ & $\begin{array}{l}\text { APR with definitely visible } \\
(\mathrm{N}=75)\end{array}$ & \\
\hline Age (year) & $60.22 \pm 10.03$ & $60.54 \pm 8.60$ & $60.07 \pm 10.68$ & 0.818 \\
\hline $\mathrm{BMI}\left(\mathrm{kg} / \mathrm{m}^{2}\right)$ & $24.33 \pm 3.14$ & $23.30 \pm 3.12$ & $24.81 \pm 3.04$ & 0.018 \\
\hline Distance of AV-APR (cm) & $9.60 \pm 1.22$ & NA & $9.60 \pm 1.22$ & NA \\
\hline Distance from seminal vesicle/uterus to rectum $(\mathrm{cm})$ & $1.28(0.85,1.81)$ & $0.70(0.52,1.07)$ & $1.55(1.13,2.00)$ & 0.018 \\
\hline Height of tumor measured by MRI (cm) & $6.89 \pm 2.53$ & $6.75 \pm 2.26$ & $6.94 \pm 2.65$ & 0.706 \\
\hline Height of tumor measured by colonoscopy $(\mathrm{cm})$ & $7.00(5.00,10.00)$ & $6.00(5.00,8.00)$ & $7.00(5.00,10.00)$ & 0.288 \\
\hline \multicolumn{5}{|l|}{ Sex } \\
\hline Male & $65(59.1 \%)$ & $22(20.0 \%)$ & $43(39.1 \%)$ & \multirow[t]{2}{*}{0.583} \\
\hline Female & $45(40.9 \%)$ & $13(11.8 \%)$ & $32(29.1 \%)$ & \\
\hline \multicolumn{5}{|l|}{ Degree of bladder filling } \\
\hline Filling & $44(40.0 \%)$ & $10(9.1 \%)$ & $34(30.9 \%)$ & 0.095 \\
\hline Not-filling & $66(60.0 \%)$ & $25(22.7 \%)$ & $41(37.3 \%)$ & NA \\
\hline \multicolumn{5}{|l|}{ Pelvic effusion } \\
\hline Yes & $19(17.3 \%)$ & $2(1.8 \%)$ & $17(15.5 \%)$ & 0.028 \\
\hline No & $91(82.7 \%)$ & $33(30.0 \%)$ & $58(52.7 \%)$ & NA \\
\hline \multicolumn{5}{|l|}{ Orientation of uterus $(\mathrm{N}=45)$} \\
\hline Anteversion & $33(73.3 \%)$ & $9(20.0 \%)$ & $24(53.3 \%)$ & 0.692 \\
\hline Retroverted & $12(26.7 \%)$ & $4(8.9 \%)$ & $8(17.8 \%)$ & NA \\
\hline \multicolumn{5}{|l|}{ T stage } \\
\hline $\mathrm{T} 1$ & $7(6.3 \%)$ & $3(2.7 \%)$ & $4(3.6 \%)$ & 0.830 \\
\hline $\mathrm{T} 2$ & $23(20.9 \%)$ & $7(6.4 \%)$ & $16(14.5 \%)$ & NA \\
\hline T3 & $79(71.8 \%)$ & $25(22.7 \%)$ & $54(49.1 \%)$ & NA \\
\hline $\mathrm{T} 4$ & $1(0.09 \%)$ & $0(0.0 \%)$ & $1(0.09 \%)$ & NA \\
\hline
\end{tabular}




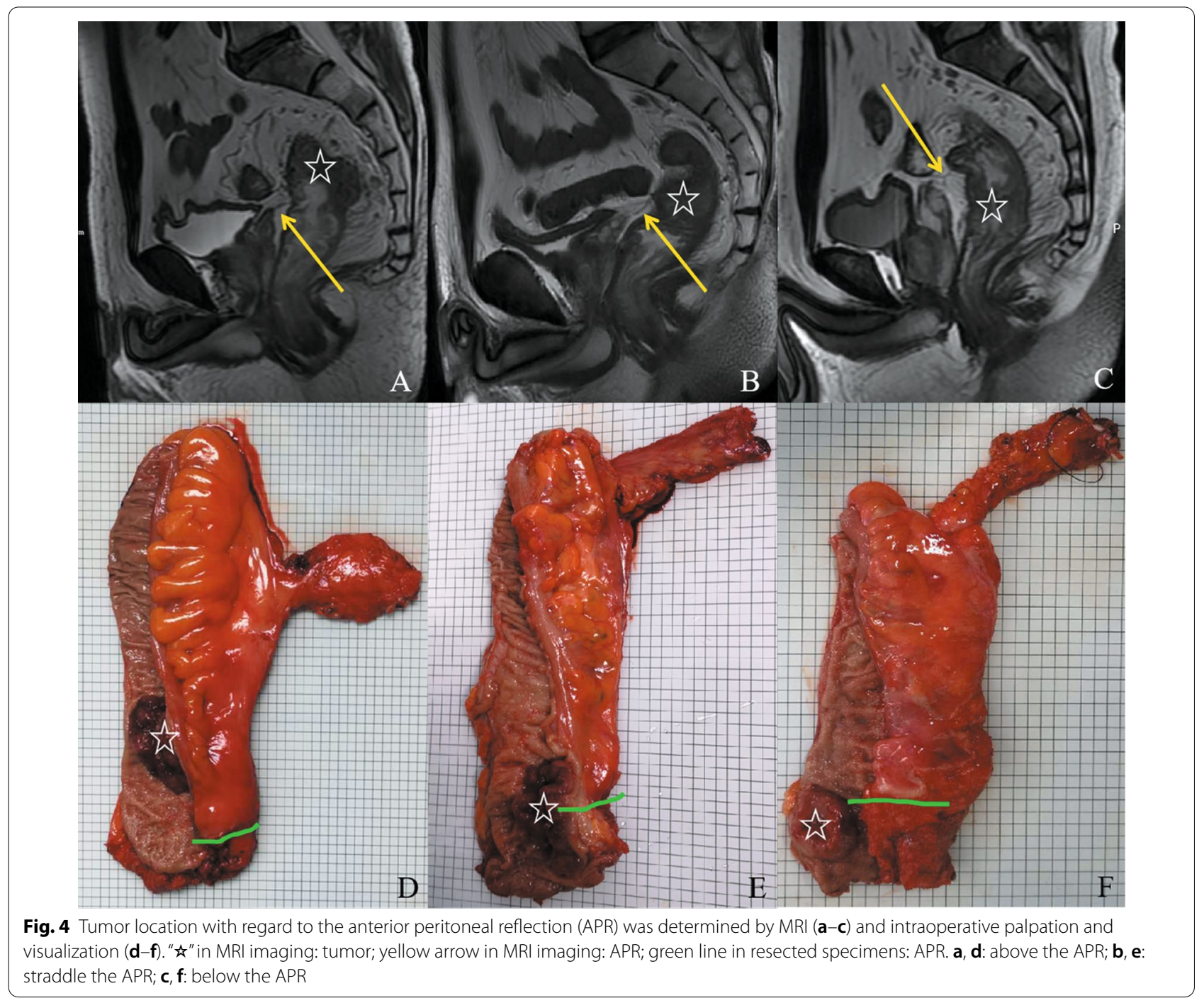

Table 2 Tumor location with regard to the APR by MRI and intraoperative findings

\begin{tabular}{lllll}
\hline & \multicolumn{2}{l}{ By intraoperative findings } & & \\
\cline { 2 - 4 } & Above the APR & Straddle the APR & Below the APR & Total \\
\hline By MRI & 18 & 2 & 3 & 23 \\
Above the APR & 0 & 34 & 6 & 40 \\
Straddle the APR & 0 & 0 & 47 & 47 \\
Below the APR & 18 & 36 & 56 & 110 \\
Total & $100 \%(18 / 18)$ & $94.40 \%(34 / 36)$ & $83.90 \%(47 / 56)$ & $90.00 \%(99 / 110)$ \\
Accuracy rate & & & &
\end{tabular}

APR, anterior peritoneal reflection

\section{Factors influencing the visualization of the APR upon MRI}

Factors influencing the visualization of the APR were BMI $(P=0.021)$, pelvic effusion $(P=0.043)$, and the distance from seminal vesicle/uterus to the rectum $(P=0.001)$, as determined by univariate analysis. Other factors, such as age, sex, $\mathrm{T}$ stage, the degree of bladder filling, the orientation of the uterus, and the location of the tumor did not influence the visualization of the APR $(P>0.05)$. 
A multivariate logistic regression analysis found that factors influencing the visualization of the APR were BMI $(P=0.031$, odds ratio, $\mathrm{OR}=1.197)$, pelvic effusion $(P=0.020, \mathrm{OR}=7.107)$ and the distance from seminal vesicle/uterus to the rectum $(P=0.001, \mathrm{OR}=3.622)$ (Table 3). The cut-off point of BMI and the distance from seminal vesicle/uterus to the rectum is $25.845 \mathrm{~kg} / \mathrm{m}^{2}$ and $1.15 \mathrm{~cm}$, respectively. We established the regression equation $\{\mathrm{Y}=-5.396+0.180 * \mathrm{BMI}+1.961 *$ [pelvic effusion (yes) $]+1.287$ * distance from seminal vesicle/uterus to the rectum 3 and the ROC curve of the combined model. The area under curve (AUC) $(95 \% \mathrm{CI})$ of the combined model is $0.840(0.750-0.930)$, the sensitivity is 0.880 and the specificity is 0.714 (Fig. 5). A predictive nomogram was constructed based on the multivariate logistical regression combined with the selected factors to develop a prediction model for the visualization of the APR (Fig. 6). The favorable calibration of the nomogram showed a non-significant Hosmer-Lemeshow test statistic $(P=0.195)$.

\section{Discussion}

In this study, we found that BMI, pelvic effusion, and the distance from seminal vesicle/uterus to rectum resulted in risk factors influencing the visualization of the APR at MRI. Sun et al. [4] found that the wider the space surrounding the APR, the easier it was to observe the APR, so we introduced the concept of the distance from seminal vesicle/uterus to the rectum, which provided the most direct reflection of the space around APR; the farther distance $(>1.15 \mathrm{~cm})$ was associated with the larger space and clearer APR display. BMI is an important indicator of the degree of obesity in the human body, which indirectly reflects the size of the pelvic space. Patients with larger BMI $\left(>25.845 \mathrm{~kg} / \mathrm{m}^{2}\right)$ had more dispersed pelvic organs as well as larger space around the APR, which made it

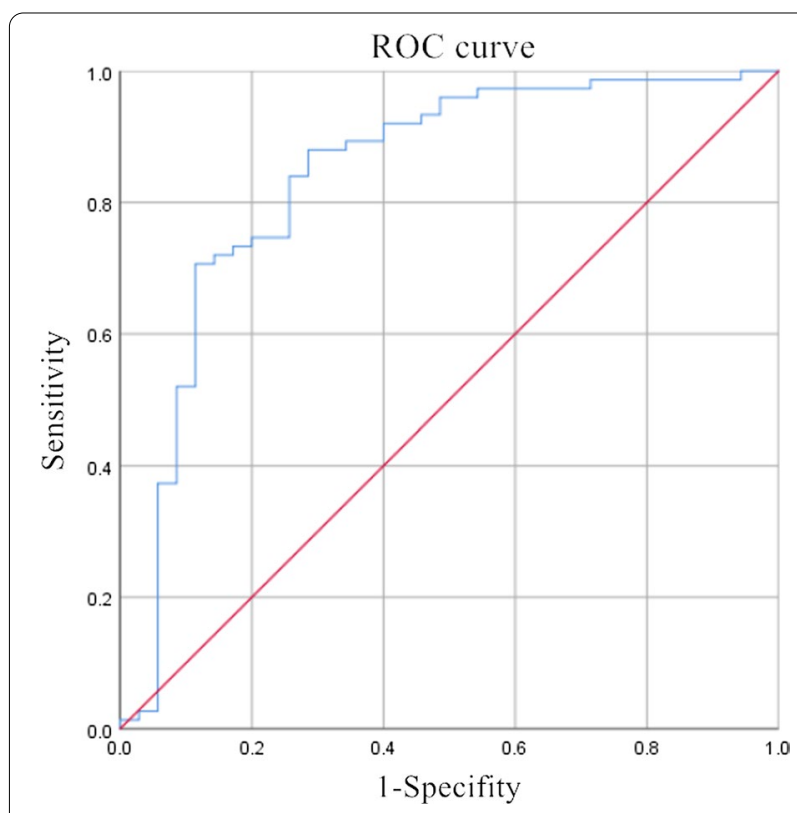

Fig. 5 The ROC curve of the combined model. The area under curve (AUC) $(95 \% \mathrm{Cl})$ of the combined model is $0.840(0.750-0.930)$, the sensitivity is 0.880 and the specificity is 0.714

easier to observe the APR. Gollub found that APR was especially visible in cases where fluid was present in the pelvic cul-de-sac [19]. We also found that in patients with pelvic effusion, APR was shown more clearly. The effusion may appear at the lowest point of the pelvic cavity, which can contrast the APR. Sun et al. [4] have reported that the degree of bladder filling, the orientation of the uterus, and age are the factors that affect the visualization of the APR (283 of 319 was visible). However, we found no association between these variables and visualization of the APR. Nevertheless, we can increase the distance from seminal vesicle/uterus to rectum by minimizing the

Table 3 Logistic regression analyses of factors that affect the visualization of the APR on MRI

\begin{tabular}{|c|c|c|c|c|}
\hline \multirow[t]{2}{*}{ Variables } & \multicolumn{2}{|c|}{ Univariate logistic regression } & \multicolumn{2}{|c|}{ Multivariate logistic regression } \\
\hline & OR $(95 \% \mathrm{Cl})$ & $P$ & OR $(95 \% \mathrm{Cl})$ & $P$ \\
\hline Age & $0.995(0.956-1.036)$ & 0.816 & NA & NA \\
\hline Sex & $1.259(0.552-2.872)$ & 0.583 & NA & NA \\
\hline T stage & $1.189(0.621-2.276)$ & 0.602 & NA & NA \\
\hline Degree of bladder filling & $0.482(0.204-1.143)$ & 0.098 & NA & NA \\
\hline Pelvic effusion & $4.836(1.051-22.250)$ & 0.043 & $7.107(1.360-37.148)$ & 0.020 \\
\hline Tumor location by intraoperative findings & $0.827(0.475-1.440)$ & 0.502 & NA & NA \\
\hline BMl & $1.192(1.026-1.385)$ & 0.021 & $1.197(1.017-1.409)$ & 0.031 \\
\hline Orientation of uterus & $0.750(0.181-3.115)$ & 0.692 & NA & NA \\
\hline Distance from seminal vesicle/uterus to rectum & $3.561(1.692-7.493)$ & 0.001 & $3.622(1.642-7.990)$ & 0.001 \\
\hline
\end{tabular}

$\mathrm{BMI}$, body mass index; $\mathrm{Cl}$, confidence interval; $\mathrm{OR}$, odd ratio 


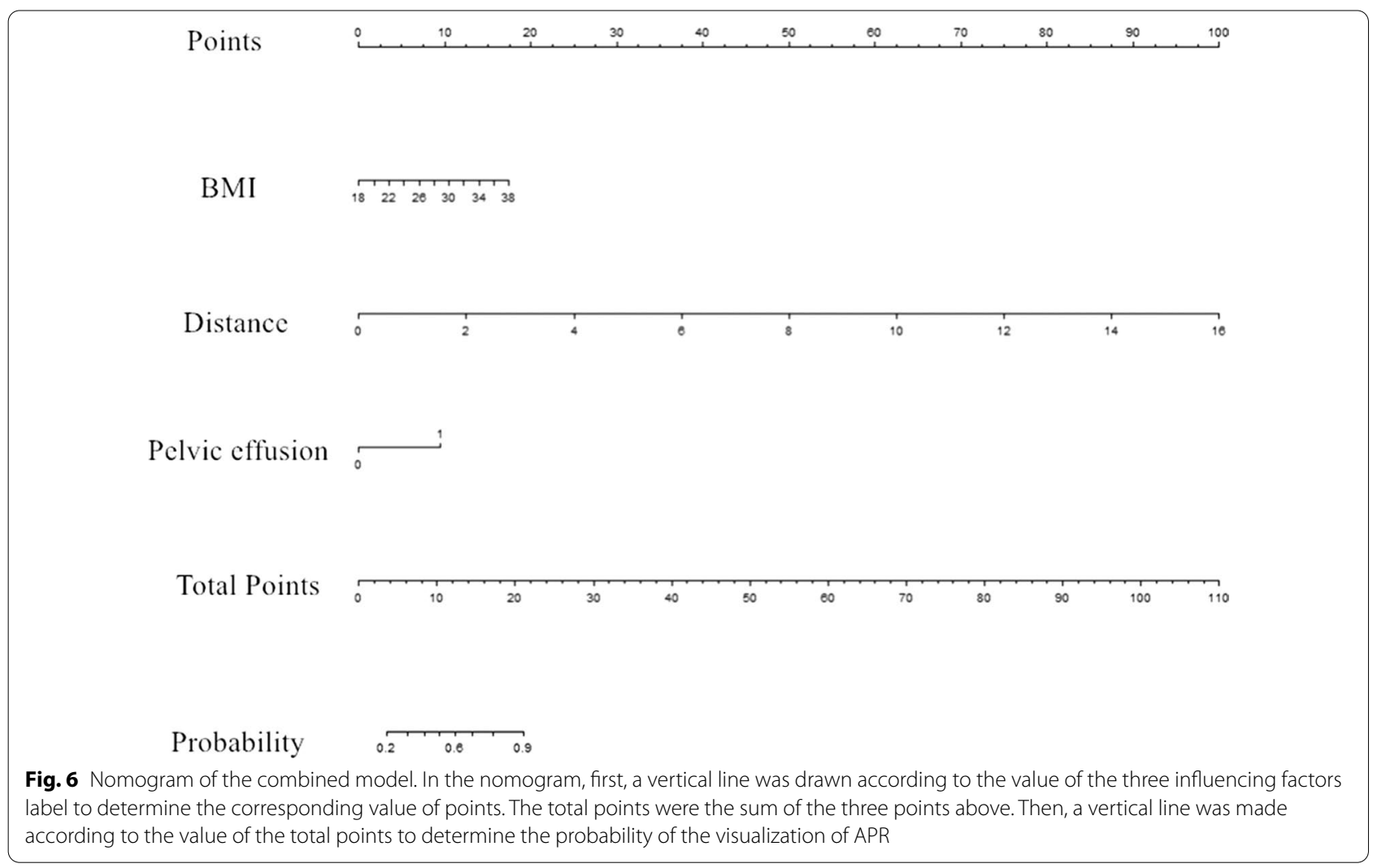

contents of the bladder, so as to improve the possibility of the visualization of the APR.

We found that the mean distance of AV-APR was $9.57 \pm 0.68 \mathrm{~cm}$ in females and $9.62 \pm 1.47 \mathrm{~cm}$ in male; the observed difference was not statistically significant, which was consistent with the results of Yun (the mean distance of AV-APR was $8.80 \pm 2.20 \mathrm{~cm}$ in females and $8.10 \pm 1.70 \mathrm{~cm}$ in male) [15]. However, Sun [4] reported the significant difference in sex, with the distance of $10.4 \pm 1.1 \mathrm{~cm}$ for females and $10.0 \pm 1.2 \mathrm{~cm}$ for males.

In this study, we also found that MRI can be used to evaluate the tumor location with regard to the APR. The accuracy was close to $90.0 \%$. In previous studies, computed tomography (CT), and transrectal ultrasonography (TRUS) were also used to evaluate the distance and location relationship between APR and tumor, but they all had different disadvantages. CT could identify the rectal cancer location with regard to the APR, but the soft tissue resolution of CT is worse than MRI, and CT examination had radiation, which was not as safe as MRI [10]. Gerdes et al. [21] recommended endorectal ultrasound (EUS) as the method of choice for predicting the location of APR. But TRUS was a practitioner-dependent subjective procedure and patients would have discomfort during the examination, which was not as simple as MRI. So, we believe that the location of a rectal tumor with regard to the APR as determined by MRI is more objective and applicable.

There are some limitations in the present study that need to be pointed out. First, due to the strict inclusion and exclusion criteria, the sample size was relatively small, so some other factors (e.g., bladder filling degree, sex and uterine position) confirmed by the previous literature were not clearly related to the visualization of the APR in this study. In the future, a large-sample study should be conducted to determine whether the above factors are correlated with the visualization of the APR. Second, MRI measurement methods currently lack standardization; thus, the generalizability of findings is uncertain. Therefore, standardized measurement and external verification are required before broadening its application. Third, we did not consider whether intraindividual differences affect the visualization of APR on MRI. This aspect, partially such as patient position and intestinal peristalsis, should be deeply discussed and considered for further research.

\section{Conclusions}

Most of the APRs are visible on MRI. Pelvic effusion, $\mathrm{BMI}$, and the distance from seminal vesicle/uterus to the rectum may influence the visualization of the APR on the MRI, which is useful for evaluating the distance of 
$\mathrm{AV}-\mathrm{APR}$, as well as the relationship between rectal cancers and the APR. This could help clinicians in choosing the appropriate clinical decisions.

\begin{abstract}
Abbreviations
APR: Anterior peritoneal reflection; AV: Anal verge; $\mathrm{BMI}$ : Body mass index; Cl: Confidence interval; CT: Computed tomography; FOV: Field of view; ICC: Intraclass correlation coefficients; MRI: Magnetic resonance imaging; OR: Odd ratio; PACS: Picture Archiving and Communication Systems; SD: Standard deviation; T stage: Tumor stage; T2Wl:T2-weighted image; TE: Echo time; TR: Repetition time; TRUS: Transrectal ultrasonography.
\end{abstract}

\section{Supplementary Information}

The online version contains supplementary material available at https://doi. org/10.1186/s12880-021-00583-7.

Additional file 1: Supplemental Table 1. Two observers' agreement for objective parameters details.

\section{Acknowledgements}

Not applicable.

\section{Authors' contributions}

$J L, X G$ and FS conceived of the present idea. MW, GY and SZ analyzed and interpreted the patient data regarding the clinical and MRI. SZ, FC and XM were major contributors in writing the manuscript. All authors read and approved the final manuscript.

\section{Funding}

This study was supported by Shanghai Pujiang Program (Grant Number: 2019PJD052), the National Natural Science Foundation of China (Grant Number: 81572332 ) and the Youth initiative fund of naval medical university (Grant Number: 2018QN05).

\section{Availability of data and materials}

The datasets used and/or analyzed during the current study are available from the corresponding author on reasonable request.

\section{Declarations}

\section{Ethics approval and consent to participate}

All methods of the present research were carried out in accordance with the Declaration of Helsinki and were approved by the local Institutional Review Board (Committee on Ethics of Biomedicine, Changhai Hospital, Shanghai, China). Informed consent was waived for this retrospective study.

\section{Consent for publication}

Not applicable.

\section{Competing interests}

The authors declare that they have no competing interests.

Received: 20 January 2021 Accepted: 8 March 2021

Published online: 17 March 2021

\section{References}

1. Bray F, Ferlay J, Soerjomataram I, Siegel RL, Torre LA, Jemal A. Global cancer statistics 2018: GLOBOCAN estimates of incidence and mortality worldwide for 36 cancers in 185 countries. CA Cancer J Clin. 2018;68:394-424.

2. Siegel RL, Miller KD, Jemal A. Cancer statistics, 2020. CA Cancer J Clin. 2020;70:7-30.

3. Chen W, Sun K, Zheng R, Zeng H, Zhang S, Xia C, et al. Cancer incidence and mortality in China, 2014. Chin J Cancer Res. 2018;30:1-12.

4. Sun Y, Tong T, Liu F, Cai S, Xin C, Gu Y, et al. Recognition of anterior peritoneal reflections and their relationship with rectal tumors using rectal magnetic resonance imaging. Medicine. 2016;95:e2889.

5. Folkesson J, Birgisson H, Pahlman L, Cedermark B, Glimelius B, Gunnarsson U. Swedish Rectal Cancer Trial: long lasting benefits from radiotherapy on survival and local recurrence rate. J Clin Oncol. 2005;23:5644-50.

6. Aschele C, Lonardi S. Multidisciplinary treatment of rectal cancer: medical oncology. Ann Oncol. 2007;18(Suppl 9):ix114-121.

7. Finlay I. Preoperative staging for rectal cancer. BMJ. 2006;333:766-7.

8. Nijkamp J, Kusters M, Beets-Tan RG, Martijn H, Beets GL, Velde CJ, et al. Three-dimensional analysis of recurrence patterns in rectal cancer: the cranial border in hypofractionated preoperative radiotherapy can be lowered. Int J Radiat Oncol Bio Phys. 2011;80:103-10.

9. Park JS, Sakai Y, Simon NS, Law WL, Kim HR, Oh JH, et al. Long-term survival and local relapse following surgery without radiotherapy for locally advanced upper rectal cancer: an international multi-institutional study. Med (Baltimore). 2016;95:e2990.

10. Revelli M, Paparo F, Bacigalupo L, Puppo C, Furnari M, Conforti C, et al. Comparison of computed tomography and magnetic resonance imaging in the discrimination of intraperitoneal and extraperitoneal rectal cancer: initial experience. Clin Imaging. 2016;40:57-62.

11. Rosenberg R. Does a rectal cancer of the upper third behave more like a colon or a rectal cancer? Dis Colon Rectum. 2010;5:761-70.

12. Bruheim K, Guren MG, Skovlund E, Hjermstad MJ, Dahl O, Frykholm G, et al. Late side effects and quality of life after radiotherapy for rectal cancer. Int J Radiat Oncol Biol Phys. 2010;76:1005-11.

13. Birgisson H, Pahlman L, Gunnarsson U, Glimelius B, Swedish Rectal Cancer Trial Group. Adverse effects of preoperative radiation therapy for rectal cancer: long-term follow-up of the Swedish Rectal Cancer Trial. J Clin Oncol. 2005;23:8697-705.

14. Nelson H, Petrelli N, Carlin A, Couture J, Fleshman J, Guillem J, et al. Guidelines 2000 for colon and rectal cancer surgery. J Natl Cancer Inst. 2001;93:583-96.

15. Yun HR, Chun HK, Lee WS, Cho YB, Yun SH, Lee WY. Intra-operative measurement of surgical lengths of the rectum and the peritoneal reflection in Korean. J Korean Med Sci. 2008;23:999-1004.

16. Najarian MM, Belzer GE, Cogbill TH, Mathiason MA. Determination of the peritoneal reflection using intraoperative proctoscopy. Dis Colon Rectum. 2004;47:2080-5.

17. Balyasnikova S, Brown G. Optimal imaging strategies for rectal cancer staging and ongoing management. Curr Treat Options Oncol. 2016;17:32

18. Bartram C, Brown G. Endorectal ultrasound and magnetic resonance imaging in rectal cancer staging. Gastroenterol Clin North Am. 2002;31:827-39.

19. Gollub MJ, Maas M, Weiser M, Beets GL, Goodman K, Berkers L, et al. Recognition of the anterior peritoneal reflection at rectal MRI. AJR Am J of Roentgenol. 2013;200:97-101.

20. Paparo F, Puppo C, Montale A, Bacigalupo L, Pascariello A, Clavarezza $M$, et al. Comparison between magnetic resonance imaging and rigid rectoscopy in the preoperative identification of intra- and extraperitoneal rectal cancer. Colorectal Dis. 2014;16:0379-385.

21. Gerdes B, Langer P, Kopp I, Bartsch D, Stinner B. Localization of the peritoneal reflection in the pelvis by endorectal ultrasound. Surg Endosc. 1998;12:1401-4.

\section{Publisher's Note}

Springer Nature remains neutral with regard to jurisdictional claims in published maps and institutional affiliations. 